\title{
Test of the hadronic interaction models SIBYLL2.3, EPOS-LHC and QGSJETII- 04 with Tibet EAS core data
}

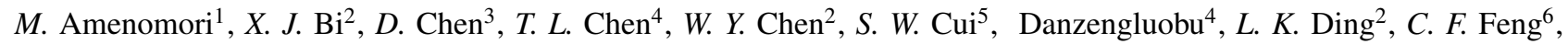

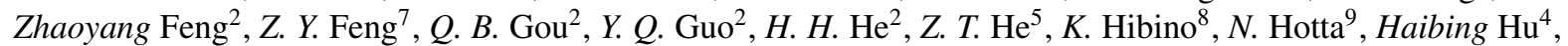
H. B. $\mathrm{Hu}^{2}, J . \mathrm{Huang}^{2}, H . Y . \mathrm{Jia}^{7}, L . \mathrm{Jiang}^{2}, F . \mathrm{Kajino}^{10}, K . \mathrm{Kasahara}^{11}, Y$. Katayose ${ }^{12}, C . \mathrm{Kato}^{13}, K . \mathrm{Kawata}^{14}, M$. Kozai $^{13,15}$, Labaciren ${ }^{4}$, G. M. Le ${ }^{16}, A . F . \mathrm{Li}^{17,6,2}, H . J . \mathrm{Li}^{4}$, W. J. Li ${ }^{2,7}, Y . H . \mathrm{Lin}^{2,18}, C . \mathrm{Liu}^{2}, J . S . \mathrm{Liu}^{2}, M . Y . \mathrm{Liu}^{4}, H . \mathrm{Lu}^{2}$, $X . R$. Meng $^{4}, T$. Miyazaki $^{13}, K$. Munakata $^{13}, T$. Nakajima $^{13}, Y$. Nakamura $^{13}, H$. Nanjo $^{1}, M$. Nishizawa $^{19}, T$. Niwa $^{13}$,

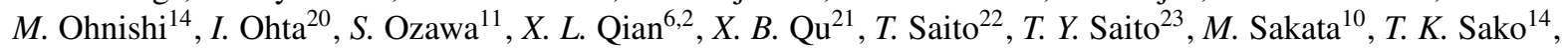

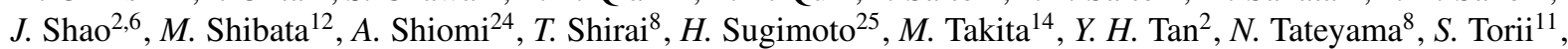
$H$. Tsuchiya ${ }^{26}, S . \mathrm{Udo}^{8}, H$. Wang ${ }^{2}, H . R . \mathrm{Wu}^{2}, L . \mathrm{Xue}^{6}, Y$. Yamamoto ${ }^{10}, K$. Yamauchi ${ }^{12}, Z$. Yang ${ }^{2}, A . F$. Yuan $^{4}, L . M$.

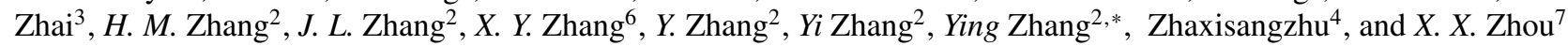
(The Tibet AS $\gamma$ Collaboration)

${ }^{1}$ Department of Physics, Hirosaki University, Hirosaki 036-8561, Japan

${ }^{2}$ Key Laboratory of Particle Astrophysics, Institute of High Energy Physics, Chinese Academy of Sciences, Beijing 100049, China

${ }^{3}$ National Astronomical Observatories, Chinese Academy of Sciences, Beijing 100012, China

${ }^{4}$ Department of Mathematics and Physics, Tibet University, Lhasa 850000, China

${ }^{5}$ Department of Physics, Hebei Normal University, Shijiazhuang 050016, China

${ }^{6}$ Department of Physics, Shandong University, Jinan 250100, China

${ }^{7}$ Institute of Modern Physics, SouthWest Jiaotong University, Chengdu 610031, China

${ }^{8}$ Faculty of Engineering, Kanagawa University, Yokohama 221-8686, Japan

${ }^{9}$ Faculty of Education, Utsunomiya University, Utsunomiya 321-8505, Japan

${ }^{10}$ Department of Physics, Konan University, Kobe 658-8501, Japan

${ }^{11}$ Research Institute for Science and Engineering, Waseda University, Tokyo 169-8555, Japan

${ }^{12}$ Faculty of Engineering, Yokohama National University, Yokohama 240-8501, Japan

${ }^{13}$ Department of Physics, Shinshu University, Matsumoto 390-8621, Japan

${ }^{14}$ Institute for Cosmic Ray Research, University of Tokyo, Kashiwa 277-8582, Japan

${ }^{15}$ Institute of Space and Astronautical Science, Japan Aerospace Exploration Agency (ISAS/JAXA), Sagamihara 252-5210, Japan

${ }^{16}$ National Center for Space Weather, China Meteorological Administration, Beijing 100081, China

${ }^{17}$ School of Information Science and Engineering, Shandong Agriculture University, Taian 271018, China

${ }^{18}$ University of Chinese Academy of Sciences, Beijing 100049, China

${ }^{19}$ National Institute of Informatics, Tokyo 101-8430, Japan

${ }^{20}$ Sakushin Gakuin University, Utsunomiya 321-3295, Japan

${ }^{21}$ College of Science, China University of Petroleum, Qingdao 266555, China

${ }^{22}$ Tokyo Metropolitan College of Industrial Technology, Tokyo 116-8523, Japan

${ }^{23}$ Max-Planck-Institut für Physik, Munich D-80805, Germany

${ }^{24}$ College of Industrial Technology, Nihon University, Narashino 275-8576, Japan

${ }^{25}$ Shonan Institute of Technology, Fujisawa 251-8511, Japan

${ }^{26}$ Japan Atomic Energy Agency, Tokai-mura 319-1195, Japan

\begin{abstract}
A hybrid experiment has been started by the AS $\gamma$ experiment at Yangbajing (4300m a.s.1.) in Tibet since May 2009, that consists of a high-energy air-shower-core array (YAC-I) and a high-density air-shower array (Tibet-III). In this paper, we report our results to check the hadronic interaction models SIBYLL2.3, SIBYLL2.1, EPOS-LHC and QGSJETII-04 in the multi-tens TeV energy region using YAC-I+Tibet-III experimental data from May 2009 through January 2010. The effective live time is calculated as 106.05 days. The results show that the description of transverse momentum, inelastic cross-section and inelasticity for the 4 hadronic interaction models is consistent with YAC-I experimental data within 15\% systematic errors range in the forward region below $100 \mathrm{TeV}$. Among them, the EPOS-LHC model is the best hadronic interaction model. Furthermore, we find that the $\mathrm{H} 4 \mathrm{a}$ composition model is the best one below the $100 \mathrm{TeV}$ energy region.
\end{abstract}

\section{Introduction}

*e-mail: yingzhang@ihep.ac.cn

Monte Carlo simulations are widely used in extensive air showers (EAS), which are based on some hadronic inter- 
action models and primary cosmic ray composition models. At present, the simulation code Corsika that is comprehensively used in the surface cosmic ray studies includes many interaction models. For accelerator experiments with energies lower than $2 \mathrm{TeV}$ - the corresponding fixed-target energy of the highest ISR energy, the inelastic interaction cross section, the interaction inelasticity and the distribution of large $\mathrm{x}$ (Feynman variable) particles (or particles produced in the forward region) have essentially been measured [1]. However, when the energy goes higher, the inelasticity and the distribution of large $\mathrm{x}$ particles produced were no longer directly measured by hadron collider experiments, and one has to use extrapolation of the laws established at lower energies. Obviously, the correctness of the extrapolation determines the correctness of the description of EASs at higher energies. For multi-parameter measurements of EASs, it seemed that no interaction model can explain all the data consistently [2]. Therefore, the hadronic interaction models need to be checked and further improved.

A hybrid experiment has been started by the AS $\gamma$ experiment at Yangbajing (4300m a.s.l.) in Tibet since May 2009, that consists of a high-energy air-shower-core array (YAC-I) and a high-density air-shower array (Tibet-III). In this paper, we investigated hadronic interaction models (QGSJETII-04, SIBYLL2.1, SIBYLL2.3 and EPOSLHC) in the forward region of EAS in the multi-tens $\mathrm{TeV}$ energy region using the data obtained by the (YACI+Tibet-III) experiment.

\section{Experiment}

A new EAS hybrid experiment called YAC (Yangbajing Air shower Core array) has been constructed near the center of the Tibet III AS array with dense spacing in Tibet, $4300 \mathrm{~m}$ above sea level (an atmosphere depth of 606 $\mathrm{g} / \mathrm{cm}^{-2}$ ). YAC-I observes high energy electromagnetic particles in air shower (AS) cores within several meters from the shower axis, and the Tibet-III array measures the total energy and the arrival direction of air showers. YAC-I detector consists of a scintillator of $40 \mathrm{~cm} \times 50 \mathrm{~cm}$ in area with $1 \mathrm{~cm}$ thickness and lead absorber of $3.5 \mathrm{~cm}$ thickness supported by a $1 \mathrm{~cm}$ thick iron plate above the scintillator. High energy electromagnetic particles near the AS axis induce local cascade showers (burst) through lead and these shower particles enter the scintillators. The scintillator is divided into 10 pieces of $5 \mathrm{~cm}$ width and a wavelength shifting fiber is used to collect the scintillation light through each piece. To achieve a wider shower size measurement under the lead layer, a wide dynamic range from 1 MIP to $10^{6}$ MIPs is demanded which is realized by two photomultipliers (PMT).

\section{Simulations}

A full Monte Carlo simulation has been carried out on the development of EAS in the atmosphere and the response in YAC-I and Tibet-III detectors. The simulation code CORSIKA (version 73500) including QGSJETII-04,
Table 1. Statistics of air-shower core events in MC simulation and experiment.

\begin{tabular}{lcc}
\hline Models & Primaries $(\mathrm{E} \geq 1 \mathrm{TeV})$ & Data set \\
\hline QGSJETII-04+He-poor & $4.86 \times 10^{10}$ & 5304 \\
SIBYLL2.1 +He-poor & $4.12 \times 10^{10}$ & 4838 \\
SIBYLL2.3 +He-poor & $2.09 \times 10^{10}$ & 2616 \\
EPOS-LHC +He-poor & $4.86 \times 10^{10}$ & 5411 \\
EPOS-LHC +He-rich & $1.27 \times 10^{12}$ & 155607 \\
EPOS-LHC +H4a & $1.74 \times 10^{12}$ & 231344 \\
\hline Expt.data & & 921 \\
\hline
\end{tabular}

SIBYLL2.1, SIBYLL2.3 and EPOS-LHC hadronic interaction models are used to generate AS events [3]. For the primary cosmic rays, we used three composition models, namely, "He-poor", "He-rich" and "H4a" models [4-6], The details of the assumed primary cosmic-ray flux are described in [7]. Primaries isotropically incident at the top of the atmosphere within zenith angles from 0 to 60 degrees are injected into the atmosphere. The minimum primary energy of this simulation is set at $1 \mathrm{TeV}$. Secondary particles are traced down to an altitude of $4300 \mathrm{~m}$ till $1 \mathrm{MeV}$. The axis of each EAS event is randomly dropped onto an area of $52.84 m \times 52.14 m$, which includes the marginal space of $25 \mathrm{~m}$ outside each side of the detectors. The electromagnetic showers in the lead layer induced by electrons or photons that hit any detector unit of the array are treated by a subroutine that is based on the detector simulation code Geant4 (version 9.5) [8]. In order to check the forward-region hadronic interaction models at multi- $\mathrm{TeV}$ energies, we selected six combinations of interaction models and primary composition models. The number of AS events generated for each model is shown in table 1 .

Normally, we can obtain the following quantities from YAC-I and Tibet-III array:

$N_{b}$ - the number of shower particles under the lead plate of a detector unit; $N_{\text {hit }}$ - the number of "fired" detector units with $N_{b} \geq$ a given threshold value; $\operatorname{sum}_{b}$ - the total burst size of all fired detector units; $N_{b}{ }^{\text {top }}-$ the maximum burst size among fired detectors; $R_{w}$ - the mean lateral spread, $\left(R_{w}=\Sigma R_{i} /\left(N_{h i t}-1\right)\right.$, where $R_{i}$ is the lateral distance from the air shower core $\left(X_{c}, Y_{c}\right)$ to the center of the $i^{\text {th }}$ fired detector; $\left\langle N_{b} R\right\rangle-$ the mean energy-flow spread, $\left\langle N_{b} R\right\rangle=\sum\left(N_{b i} \times r_{i}\right) / N_{h i t} ; N_{e}$ - the air shower size is estimated by fitting the lateral density distribution using the NKG function; $\theta$ - the arrival direction of the air shower.

In this paper, we obtain the samples under the following conditions: $N_{b} \geq 200, N_{h i t} \geq 3, N_{b}^{\text {top }} \geq 500$, $8 \times 10^{4}<N_{e}<10^{5}$, the mode energy as known from the Monte Carlo is about $80 \mathrm{TeV}$. We used the experimental data set obtained from May 2009 through January 2010. The data sample coming from successful coincidence corresponds a live time of 106.05 days. The simulated data were analyzed in the same manner as in the procedure for the experimental data analysis. The statistics of core events of two data sets in MC simulation and experiment are listed in table 1. 


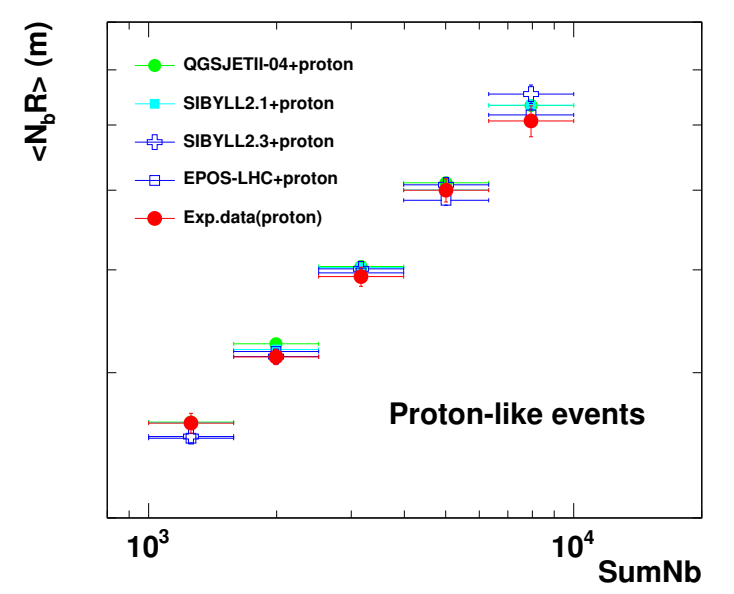

Figure 1. Below the $100 \mathrm{TeV}$ energy region, using pure-protons YAC-I experimental data to check the distribution of the $\left\langle N_{b} R\right\rangle$ parameter.

\section{Results and Discussion}

\subsection{Check transverse momentum}

In order to avoid the "ambiguous" interference of the cosmic-ray components, we selected the proton events by ANN (Artificial Neural Network) from experimental data in the same way as for simulation data, whose applicability to our experiment was well confirmed by the MC simulation. In this ANN analysis, we use the following seven quantities:(1) $N_{\text {hit }}$, (2) $\operatorname{sum}_{b}$, (3) $N_{b}{ }^{\text {top }}$, (4) $R_{w}$, (5) $\left\langle N_{b} R\right\rangle$, (6) $N_{e}$, (7) $\theta$. These are input to the ANN with 35 hidden nodes and 1 output unit. To train the ANN in separating protons from other nuclei, the input patterns for proton and other-nuclei are set to 0 and 1 , respectively. We then define a critical value of $T_{c}$ to calculate the corresponding purity of the selected Proton-like events. In this paper, events with $T_{c}<0.2$ are regarded as Proton-like events, and the average selection purity over the whole energy range of Proton-like events is $\sim 85 \%$.

Due to the selected Proton-like events, we can avoid the "ambiguous" interference of the cosmic-ray components and use some weighted average lateral spread of the secondary particles $\left(\left\langle N_{b} R\right\rangle\right)$ observed by the YAC to test the transverse momentum in the hadronic interaction models. Figure 1 shows the correlation between $\operatorname{sum}_{b}$ and $\left\langle N_{b} R\right\rangle$ for various hadronic interaction models using Proton-like simulation data and YAC-I experimental data. It can be seen that the YAC-I experimental data is compatible with the distribution of NbR based on EPOS-LHC, QGSJETII-04, SIBYLL2.1 and SIBYLL2.3 using Protonlike events. Especially, YAC-experimental data are very close to EPOS-LHC. After excluding the "ambiguous" interference of cosmic-ray components, we can obtain the following conclusions: (1) The transverse momentum description of EPOS-LHC, QGSJETII-04, SIBYLL2.1 and SIBYLL2.3 is consistent with YAC within $15 \%$ systematic errors in the forward region below the $100 \mathrm{TeV}$ energy region; (2) Among them, the EPOS-LHC model is

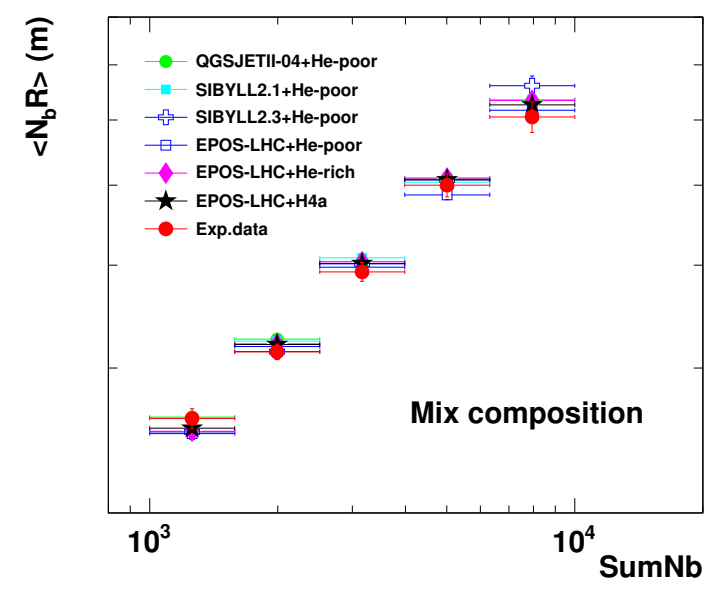

Figure 2. Below the $100 \mathrm{TeV}$ energy region, using the allparticle YAC -I experimental data to check primary composition models.

the most consistent with YAC-I experimental results for the description of transverse momentum.

\subsection{Check primary composition model}

After we get the best ineraction models for the description of transverse momentum, we can compare 6 sets of simulation data combining the cosmic-ray composition models and hadronic interaction models (QGSJETII-04+Hepoor, SIBYLL2.1+He-poor, SIBYLL2.3+He-poor, EPOS-LHC+He-poor, EPOS-LHC+He-rich and EPOS$\mathrm{LHC}+\mathrm{H} 4 \mathrm{a}$ ) with YAC-I experimental data, as shown in figure 2. One can see that the experimental data is consistent with simulation data under different cosmic-ray composition models and hadronic interaction models. From the above section, we know that the EPOS-LHC model best describes the transverse momentum in the forward region. Based on this, we find that the EPOS-LHC+H4a model is most consistent with YAC-I experimental results. As we know, the EPOS-LHC model is the most consistent for the description of transverse momentum, so we can get the following conclusions: (1) H4a composition model is most consistent with YAC-I experimental results below $100 \mathrm{TeV}$ energy region; (2) We think that the $\mathrm{H} 4 \mathrm{a}$ composition model is reliable below $100 \mathrm{TeV}$ because the proton and helium spectrum of the $\mathrm{H} 4 \mathrm{a}$ model coincides with the latest results from CREAM3.

\subsection{Check inelastic cross section and inelasticity}

It is well known that the absolute intensity of the total burst sizes depends sensitively on the increase of cross sections, inelasticity, and also on the primary cosmic-ray composition. From the above section, we know that the H4a composition model is the best one below $100 \mathrm{TeV}$. Therefore, the absolute flux of the secondary particles (sumNb) can be used to test the inelastic cross section 

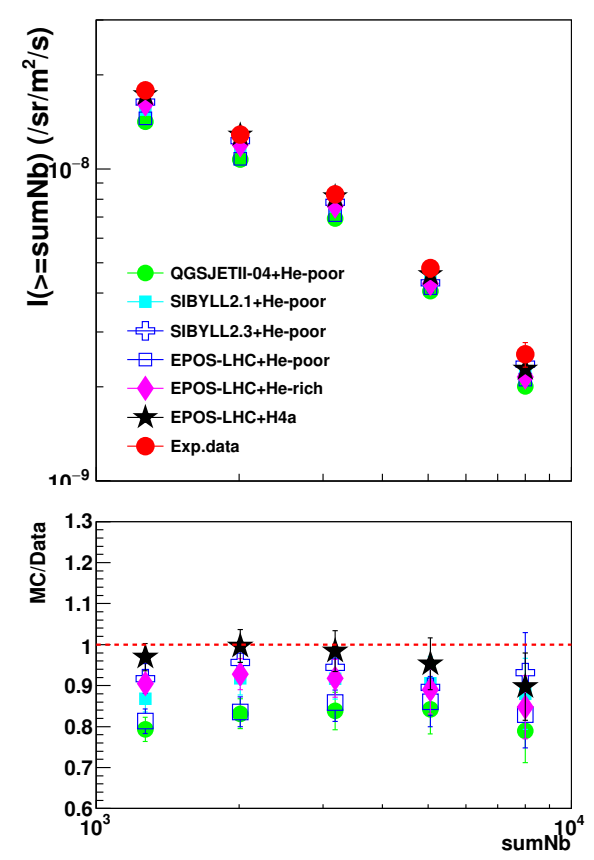

Figure 3. Below $100 \mathrm{TeV}$ energy region, Check of the inelastic cross section of hadronic interaction models using YAC-I experimental data.

and inelasticity in the hadronic interaction models. We compare 6 sets of simulation data combining the cosmic-ray composition models and hadronic interaction models (QGSJETII-04+He-poor, SIBYLL2.1+He-poor, SIBYLL2.3+He-poor, EPOS-LHC+He-poor, EPOS$\mathrm{LHC}+\mathrm{He}$-rich and EPOS-LHC+H4a) with YAC-I experimental data, as shown in figure 3 . The top figure is the comparison of the integral total burst-size spectrum $\left(\operatorname{sum}_{b}\right)$ between MC and YAC-I experimental data. The bottom figure is the intensity ratios of $\operatorname{sumN}_{b}$ to that obtained by the experimental data, the red dash line $($ Ratio $=1)$ denotes the experimental data. We can see that EPOS-LHC+H4a is most consistent with YAC-I data below the $100 \mathrm{TeV}$ energy region. From the above section, we know that the H4a composition model is the best one below the $100 \mathrm{TeV}$ energy region. Based on this, we can conclude that EPOS-LHC best describes the inelastic cross section and inelasticity in the forward region. And the inelastic cross section and inelasticity description of EPOS-LHC, QGSJETII-04, SIBYLL2.1 and SIBYLL2.3 is consistent with YAC within $15 \%$ systematic errors.

\section{Summary}

In this paper, we checked SIBYLL2.3, SIBYLL2.1, EPOS-LHC and QGSJETII-04 hadronic interaction models in the forward region below the $100 \mathrm{TeV}$ energy region by using the events observed simultaneously by YACI+Tibet-III and applying a neural network analysis (ANN). We find that the description of transverse momentum, inelastic cross-section and inelasticity for the 4 hadronic interaction models is correct within $15 \%$ systematic errors range in the forward region below the $100 \mathrm{TeV}$ energy region. Among them, the EPOS-LHC model is the best hadronic interaction model. Furthermore, we find that the H4a composition model is the best one below the $100 \mathrm{TeV}$ energy region. A new hybrid experiment (YACII+Tibet-III+MD) has been constructed and started taking data since 2014 and more results will be expected.

\section{Acknowledgements}

The collaborative experiment of the Tibet Air Shower Arrays has been performed under the auspices of the Ministry of Science and Technology of China and the Ministry of Foreign Affairs of Japan. This work is supported by the National Key R\&D Program of China (No. 2016YFE0125500), by the Grants from the National Natural Science Foundation of China (Nos.11803038,11533007,11673041,11603047 and 11275212), and by the Key Laboratory of Particle Astrophysics, Institute of High Energy Physics, CAS. This work is supported by the joint research program of the Institute for Cosmic Ray Research (ICRR), The University of Tokyo.

\section{References}

[1] M. Albrow, A. Bagchus, D. Barber, et al, Nuclear Physics B 73, 40 (1974)

[2] O. Adriani, L. Bonechi, M. Bongi, et al, Physics Letters B 703, 128 (2011)

[3] D. Heck, J. Knapp, J.N. Capdevielle, et al, Tech. rep. (1998)

[4] M. Amenomori, et al, The Astrophysical Journal 678, 1165 (2008)

[5] M. Shibata, Y. Katayose, J. Huang, D. Chen, The Astrophysical Journal 716, 1076 (2010)

[6] T.K. Gaisser, Astroparticle Physics 35, 801 (2012)

[7] L.M. Zhai, J. Huang, D. Chen, et al, Journal of Physics G: Nuclear and Particle Physics 42, 045201 (2015)

[8] S. Agostinelli, J. Allison, K. Amako, et al, Nuclear Instruments and Methods in Physics Research, Section A: Accelerators, Spectrometers, Detectors and Associated Equipment 506, 250 (2003), 1005 . 0727v1 